\title{
PERFIL DE PACIENTES COM INCONTINÊNCIA URINÁRIA EM UM AMBULATÓRIO DE HOSPITAL UNIVERSITÁRIO
}

\author{
Francisca das Chagas Sheyla Almeida Gomes Braga1,* (0), Claudia Daniella Avelino Vasconcelos

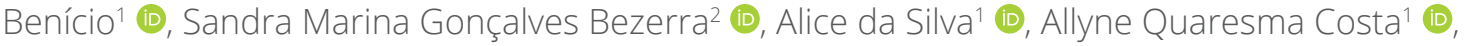

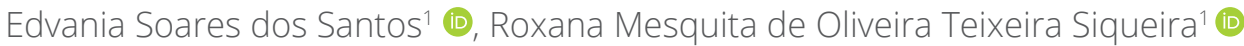

\begin{abstract}
RESUMO
Objetivo: conhecer o perfil sociodemográfico e clínico das pessoas com incontinência urinária em ambulatório de Hospital Universitário. Métodos: estudo transversal, em que se utilizou formulário semiestruturado com 63 mulheres que aguardavam atendimento nos ambulatórios de ginecologia e urologia, entre os meses de julho e agosto de 2019. Os dados foram processados no software Statistical Package for the Social Sciences - SPSS 20.0. Resultados: foram realizadas entrevistas com mulheres com idade entre 19 e 77 anos. Dessas, 55,6\% mantinha vida sexual ativa; 85,7\% apresentava doença preexistente; 69,8\% presentou perda urinária ao tossir, espirrar sorrir, colocar peso e/ou fazer esforços; $12,7 \%$ precisa urinar assim que sentem vontade; e 17,5\% apresenta perda de urina nas duas situações. Cerca de $35 \%$ procurou consulta devido à bexiga baixa e/ou perda de urina; $27 \%$ teve de 4 a 5 gestações. Dentre os fatores de risco associado à incontinência urinária, 28,6\% tinha diabetes mellitus, $61,9 \%$ hipertensão arterial, 82,5\% fazia uso de medicação contínua, 11,1\% era obesa, 34,9\% relatou problemas de constipação e 74,6\% fez cirurgias pélvicas. Conclusão: concluiu-se que todas as mulheres do estudo apresentaram mais de um fator de risco para desenvolver incontinência urinária, reforçando sua multifatorialidade, bem como a importância de um tratamento holístico e multiprofissional.
\end{abstract}

DESCRITORES: Enfermagem. Incontinência urinária. Perfil de saúde. Prevalência. Estomaterapia.

\section{PROFILE OF PATIENTS WITH URINARY INCONTINENCE IN A UNIVERSITY HOSPITAL OUTPATIENT CLINIC}

\begin{abstract}
Objective: to know the sociodemographic and clinical profile of people with urinary incontinence in an outpatient clinic of a University Hospital. Methods: cross-sectional study, using a semi-structured form with 63 women who were awaiting care at the gynecology and urology outpatient clinics, between the months of July and August 2019. The data were processed in the software Statistical Package for the Social Sciences - SPSS 20.0. Results: interviews were carried out with women aged between 19 and 77 years. Of these, $55.6 \%$ maintained an active sex life; $85.7 \%$ had pre-existing disease; $69.8 \%$ presented urinary loss when coughing, sneezing, smiling, putting on weight and/or making efforts; $12.7 \%$ need to urinate as soon as they feel like it; and $17.5 \%$ had loss of urine in both situations. About 35\% sought consultation due to low bladder and/or loss of urine; $27 \%$ had 4 to 5 pregnancies. Among the risk factors associated with urinary incontinence, $28.6 \%$ had diabetes mellitus, $61.9 \%$ had arterial hypertension, $82.5 \%$ used continuous medication, $11.1 \%$ was obese, $34.9 \%$ reported constipation problems and
\end{abstract}

1. Universidade Federal do Piauí - Centro de Ciências da Saúde - Departamento de Enfermagem - Teresina (PI), Brasil. 2. Universidade Estadual do Piauí - Centro de Ciências da Saúde - Departamento de Enfermagem - Teresina (PI), Brasil. *Autora correspondente: sheylagomesbraga@gmail.com Editor de Seção: Gisela Maria Assis

Recebido: Dez. 14, 2020 | Aceito: Fev. 20, 2021

Como citar: Braga FCSAG; Benício CDAV; Bezerra SMG; Silva A; Costa AQ; Santos ES; Siqueira RMOT. Perfil de pacientes com incontinência urinária em um ambulatório de hospital universitário. ESTIMA, Braz. J. Enterostomal Ther., 2021, 19 : e0721. https://doi.org/10.30886/estima.v19.997_PT 
74.6\% underwent pelvic surgery. Conclusion: it was concluded that all women in the study had more than one risk factor for developing urinary incontinence, reinforcing their multifactoriality, as well as the importance of a holistic and multiprofessional treatment.

DESCRIPTORS: Nursing. Urinary incontinence. Health profile. Prevalence. Stomatherapy.

\title{
PERFIL DE LOS PACIENTES CON INCONTINENCIA URINARIA EN UNA CLÍNICA AMBULATORIA DE UN HOSPITAL UNIVERSITÁRIO
}

\begin{abstract}
RESUMEN
Objetivo: Conocer el perfil sociodemográfico y clínico de personas con incontinencia urinaria en una consulta externa de un Hospital Universitario. Métodos: El estudio transversal, se utilizó un formulario semiestructurado con 63 mujeres que se encontraban en espera de atención en las consultas externas de Ginecología y Urología, entre los meses de julio y agosto de 2019. Los datos se procesaron mediante el Paquete Estadístico de la Ciencias sociales: software SPSS. Resultados: Se realizaron entrevistas con mujeres,; edad entre 19 y 77 años; mantuvieron una vida sexual activa 55,6\% y 85,7\% tenían enfermedad preexistente. Presentaron pérdidas urinarias al toser, estornudar, sonreír, engordar y/o realizar esfuerzos 69,8\%; El 12,7\% necesita orinar tan pronto como le apetece y el 17,5\% tiene pérdida de orina en ambas situaciones. Aproximadamente el 35\% buscó consulta debido a la disminución de la vejiga y / o la pérdida de orina; El 27\% tuvo de 4 a 5 embarazos. Entre los factores de riesgo asociados a la incontinencia urinaria, el 28,6\% tenía diabetes mellitus, el 61,9\% hipertensión arterial, el 82,5\% usaba medicación continua; El 11,1\% eran obesos; 34,9\% informó problemas de estreñimiento; El 74,6\% se sometió a cirugía pélvica. Conclusión: Se concluye que todas las mujeres del estudio tenían más de un factor de riesgo para desarrollar IU, reforzando su multifactorialidad, así como la importancia de un tratamiento holístico y multiprofesional.
\end{abstract}

DESCRIPTORES: Enfermería. Incontinencia urinaria. Perfil de salud. Prevalencia. Estomaterapia.

\section{INTRODUÇÃO}

A International Continence Society (ICS) define incontinência urinária (IU) como a perda involuntária de urina. Embora considerada uma questão prioritária de saúde, a IU é subnotificada e subdiagnosticada, tendo um baixo reconhecimento profissional. Em 1998 passou a fazer parte da Classificação Internacional de Doenças (CID), deixando de ser considerada apenas um sintoma e passando a ser uma doença ${ }^{1}$.

Segundo a Sociedade Brasileira de Urologia (SBU), essa condição acomete cerca de 400 milhões de pessoas no mundo, e no Brasil há em média 10 milhões de pessoas com incontinência ${ }^{2}$. Mesmo sendo mais comum em mulheres, atinge a população, não importando sexo, etnia ou idade. Apresenta prevalência crescente, conforme aumento da idade populaciona ${ }^{3-5}$.

Entre os estudos populacionais gerais, a prevalência da IU variou entre $25 \%$ e $45 \%$ em mulheres adultas, e em homens adultos houve publicações com variações entre 1\% e 39\%, sendo que a proporção em mulheres é, pelo menos, duas vezes maior quando comparada com os homens, ou seja, de 2:1. Não existe padronização de ferramentas e métodos empregados nos estudos de prevalência que defina IU na prática, o que pode tornar ampla a variação da prevalência, que ainda é pouco estabelecida ${ }^{6}$.

No Brasil, são poucos os estudos sobre prevalência de IU, sendo a maioria voltado apenas para mulheres. Dentre os estudos encontrados, um realizado com adultos da população urbana de Pouso Alegre, Minas Gerais, concluiu que 20,1\% da população geral apresenta IU. Entre mulheres e homens, a prevalência foi de 32,9\% e 6,2\%, respectivamente, tendo uma proporção de aproximadamente 5:17.

Já no Piauí não é conhecida a prevalência da IU, entretanto um estudo realizado em 2016 com mulheres na atenção básica em Teresina, mostrou que das 306 participantes do estudo, 40,8\% (125) eram incontinentes ${ }^{8}$. 
Corroborando os estudos supracitados, o Consenso Nacional sobre Uroginecologia de 2018 apresentou a prevalência dos tipos mais comuns de IU, em que a incontinência urinária de esforço (IUE) tem uma prevalência de 10 a 39\%, sendo a que apresenta maior taxa, seguida pela incontinência urinária mista (IUM) com 7,5 a 25\%. A incontinência urinária de urgência (IUU), isolada, é a menos frequente e corresponde de 1 a $7 \%$. As demais causas de incontinência têm uma prevalência estimada de 1 a $7 \%{ }^{2}$.

A IU tem profundo impacto negativo no bem-estar e na qualidade de vida das pessoas acometidas, o que leva, muitas vezes, a um constrangimento social, redução de emprego, diminuição da produtividade no trabalho, afeta a vida sexual, além de apresentar uma significativa carga econômica e na saúde 6 .

As pessoas com IU se beneficiam quando expostas a tratamento específico por meio de intervenções de enfermagem, contudo a maioria das pessoas desconhece essa informação por ser uma disfunção pouco divulgada na área da saúde e nos meios de comunicação, o que dificulta o esclarecimento e a identificação pela população de tal condição9.

Assim, conhecendo a alta prevalência da IU em todo o mundo, bem como pelo interesse em identificar os principais aspectos das pessoas com IU atendidas em instituição pública do Piauí, a fim de desenvolver ações junto à população do Estado, surgiu o seguinte questionamento: qual o perfil sociodemográfico e clínico das pessoas com IU atendidas nos ambulatórios de urologia e ginecologia do Hospital Universitário?

Dessa forma, o estudo objetivou caracterizar o perfil sociodemográfico e clínico das pessoas com IU atendidas nos ambulatórios de ginecologia e urologia de Hospital Universitário. Esses dados ampararam a elaboração de tecnologia educacional para adultos e contribuirá para o desenvolvimento de estratégias de cuidados a serem prestados à população do Estado, ao mesmo tempo em que subsidiará a construção de protocolo institucional para atendimento ambulatorial de pessoas com IU.

\section{MÉTODO}

Estudo descritivo, analítico e transversal a respeito da caracterização do perfil dos pacientes com IU atendidos em ambulatório de Hospital Universitário localizado em Teresina, PI. Dentre as especialidades existentes, a instituição dispõe dos serviços de urologia e ginecologia, bem como ambulatório de estomaterapia e demais especialidades multiprofissionais.

O serviço de urologia realiza exames de urofluxometria e estudo urodinâmico, consultas e cirurgias. Em 2018 foram realizadas em torno de 156 consultas por mês, perfazendo 1.874 atendimentos por ano. Já o serviço de ginecologia ocorre no ambulatório de saúde da mulher, onde são realizados consultas e exames de colpocitologia oncótica, colposcopia, histeroscopia e avaliação urodinâmica, além de cirurgias. Em 2018 foram realizados cerca de 602 atendimentos por mês, perfazendo 7.224 por ano.

A população foi constituída por pacientes atendidos no ambulatório pelas especialidades de ginecologia e urologia. A amostra foi determinada pelos seguintes critérios de inclusão: homens e mulheres que aguardavam consulta para as especialidades de ginecologia ou urologia, com idade a partir de 18 anos no momento da entrevista, apresentando queixa de perda involuntária de urina. Foram utilizados como critério de exclusão: usuários que aguardavam atendimento sem queixa de perda urinária e pessoas com deficiência cognitiva que inviabilizasse responder ao formulário.

Foram abordados 339 pacientes que aguardavam consultas nos serviços de ginecologia e urologia. Desses, 76 relataram apresentar sintomas de IU, porém 13 pessoas se recusaram a participar. Assim, a amostra constituiu-se por 63 participantes no total.

Os dados foram coletados por meio de um formulário semiestruturado, previamente validado por Benício $(2018)^{10}$ e Favoretto $(2015)^{11}$, e adaptado com questões concernentes aos objetivos propostos, contendo informações acerca das características sociodemográficas, econômica, condição clínica e fatores de risco relacionados ao tema. As coletas ocorreram nos turnos da manhã e da tarde, entre os meses de julho e agosto de 2019. Os entrevistados responderam verbalmente às perguntas do pesquisador sem que houvesse interferência em suas repostas. 
Para a realização das entrevistas, os participantes foram, inicialmente, abordados sobre a existência ou não de incontinência. Em caso positivo, o paciente era convidado a participar da pesquisa, sendo realizadas as formalizações, explicando os objetivos, os riscos e benefícios. Em caso de concordância, os participantes eram conduzidos a um espaço reservado, como consultórios ou sala de espera. Em seguida, recebiam mais informações sobre o estudo e, os que aceitavam participar, assinavam o Termo de Consentimento Livre e Esclarecido.

Os dados foram submetidos a processo de digitação, utilizando planilhas do aplicativo Microsoft ${ }^{\circledR}$ Excel ${ }^{\circledR}$ versão 16.0 e posteriormente exportados para Statistical Package for the Social Sciences - SPSS versão 20.0. O perfil sociodemográfico, clínico e os fatores de risco associados foram apresentados em tabelas e descritos por meio de frequências absolutas e percentuais. Alguns dados clínicos também foram delineados por meio das estatísticas descritivas: média e desvio padrão.

O estudo respeitou as diretrizes da Resolução no 466/2012 do Conselho Nacional de Saúde (CNS), que trata das pesquisas envolvendo seres humanos, preservando o anonimato, a privacidade e a confidencialidade dos envolvidos. O estudo faz parte de um macroprojeto intitulado "Boas práticas no cuidar de enfermagem ao paciente hospitalizado: tecnologias para mensurar, implementar e avaliar”, o qual foi submetido ao Comitê de Ética em Pesquisa (CEP) da Universidade Federal do Piauí (UFPI), sob o CAAE 01564818.2.0000.5214 e aprovado pelo parecer de no 3.305.182.

\section{RESULTADOS}

\section{Perfil sociodemográfico}

Analisando o perfil sociodemográfico dos 63 participantes, todos eram do sexo feminino; com idade média de $52,1 \pm 13,4$ anos; sendo a maioria de cor parda $(77,8 \%)$, seguida pela cor negra $(9,5 \%)$ e branca $(7,9 \%)$. Quanto à escolaridade, a maior parte tinha ensino fundamental incompleto (39,7\%); em relação à ocupação, 30,2\% possuía um emprego, 27\% era aposentada; e 12,7\% não realizava nenhuma atividade; com renda individual ou familiar de até 1 salário mínimo era 55,6\%; dentre as que possuíam renda de até 1 salário mínimo, $43 \%$ sustentava 4 pessoas. Quando questionadas sobre a situação conjugal, a maioria era casada (63,5\%), e as demais viviam em união estável ou eram solteiras, ambas as situações com 11,1\%. Dados dispostos na Tabela 1.

Tabela 1. Perfil sociodemográfico dos participantes com incontinência urinária atendidos em ambulatório de um Hospital Universitário (n=63). Teresina (PI), Brasil - 2019.

\begin{tabular}{lc}
\hline Variáveis & $\mathbf{n}(\%)$ \\
\hline Sexo & $0(0)$ \\
\hline Masculino & $63(100)$ \\
\hline Feminino & $49(77,8)$ \\
\hline Cor & $6(9,5)$ \\
\hline Parda & $5(7,9)$ \\
\hline Negra & $3(4,8)$ \\
\hline Branca & $2(3,2)$ \\
\hline Outra & $25(39,7)$ \\
\hline Escolaridade & $9(14,2)$ \\
\hline Não alfabetizado & $3(4,8)$ \\
\hline Ensino fundamental incompleto & $17(27)$ \\
\hline Ensino fundamental completo & $2(3,2)$ \\
\hline Ensino médio incompleto & $5(7,9)$ \\
\hline Ensino médio completo & \\
\hline Ensino superior incompleto & \\
\hline Ensino superior completo & \\
\hline
\end{tabular}


Tabela 1. Continuação...

\begin{tabular}{|c|c|}
\hline Variáveis & n (\%) \\
\hline \multicolumn{2}{|l|}{ Ocupação } \\
\hline Não realiza nenhuma atividade & $8(12,7)$ \\
\hline Aposentada & $17(27)$ \\
\hline Dona de casa & $14(22,2)$ \\
\hline Trabalhadora rural & $5(7,9)$ \\
\hline Emprego formal & $19(30,2)$ \\
\hline \multicolumn{2}{|l|}{ Renda } \\
\hline Até 1 salário mínimo & $35(55,6)$ \\
\hline Entre 1 e 2 salários mínimos & $14(22,2)$ \\
\hline Entre 2 e 3 salários mínimos & $9(14,3)$ \\
\hline Entre 3 e 4 salários mínimos & $5(7,9)$ \\
\hline \multicolumn{2}{|l|}{ Número de dependentes } \\
\hline Um & $7(11,1)$ \\
\hline Dois & $16(25,4)$ \\
\hline Três & $15(23,8)$ \\
\hline Quatro & $10(15,9)$ \\
\hline Cinco & $4(6,3)$ \\
\hline Mais de cinco & $11(17,5)$ \\
\hline \multicolumn{2}{|l|}{ Situação conjugal } \\
\hline Solteira & $7(11,1)$ \\
\hline Casada & $40(63,5)$ \\
\hline Viúva & $6(9,5)$ \\
\hline Separada & $3(4,8)$ \\
\hline União estável & $7(11,1)$ \\
\hline TOTAL & $63(100)$ \\
\hline
\end{tabular}

\section{Perfil clínico}

De acordo com a Tabela 2, no tópico relativo à vida sexual ativa, 55,6\% mantinha vida sexual ativa e 44,4\% não mantinha relações sexuais. Dessas, 55,6\% relatou que a IU não interferia na vida sexual e 6,3\% relatou que interferia. Sobre a existência de alguma doença preexistente, 85,7\% apresentou alguma doença além da incontinência.

Quando questionado sobre o tempo em que vem apresentando quadro de perda de urina, foi possível observar um tempo médio de 5,8 \pm 9,5 anos, o menor tempo relatado foi de 0,16 anos ( 2 meses) e o maior de 66 anos, o tempo mediano foi de 3 anos, ou seja, 50\% começou a perder urina há 3 anos.

Em relação à perda de urina, o maior número de entrevistados, $69,8 \%$, relatou apresentar perda ao tossir, espirrar, sorrir, colocar peso e/ou fazer esforço; $12,7 \%$ precisa fazer xixi assim que sente vontade, se não faz na roupa; e 17,5\% apresenta perda de urina nas duas situações anteriores.

No relato dos participantes a respeito de sua queixa principal para a realização da consulta naquele dia no serviço, destacou-se bexiga baixa e/ou perda de urina, com 34,9\%, seguido de marcação de cirurgia ou retorno pós-cirúrgico, com $17,5 \%$.

Quanto aos antecedentes ginecológicos, 41,3\% teve de 1 a 3 gestações; 20,6\% mais de 6 gestações; e 11,1 \% nunca engravidou. No que concerne à ocorrência de aborto, 39,7\% relatou ter tido ao menos um caso de abortamento.

No quesito número e tipo de parto por pessoa, observa-se que, do total de participantes, $11,1 \%$ não teve parto; 31,7\% teve de 1 a 3 partos normais e nenhum cesáreo; 19\% teve de 4 a 6 partos normais e nenhum cesáreo; 7,9\% 
teve 7 ou mais partos normais e nenhum cesáreo; 15,9\% teve de 1 a 3 partos normais e 1 cesáreo; 4,8\% teve de 4 a 6 partos normais e 1 cesáreo; 1,6\% teve 7 partos normais e 1 cesáreo; 4,8\% teve de 1 a 3 partos normais e 2 cesáreos; e $4,8 \%$ teve de 1 a 3 partos normais e 3 cesáreos.

Tabela 2. Perfil clínico e antecedentes ginecológicos das participantes com incontinência urinária atendidas em um Hospital Universitário (n=63). Teresina (PI), Brasil - 2019.

\begin{tabular}{|c|c|}
\hline Variáveis & $\mathrm{n}(\%)$ \\
\hline \multicolumn{2}{|l|}{ Vida sexual ativa } \\
\hline $\operatorname{sim}$ & $35(55,6)$ \\
\hline Não & $28(44,4)$ \\
\hline \multicolumn{2}{|l|}{ Incontinência atrapalha a vida sexual } \\
\hline Sim & $4(6,3)$ \\
\hline Não & $35(55,6)$ \\
\hline Não se aplica & $24(38,1)$ \\
\hline \multicolumn{2}{|l|}{ Apresenta alguma doença associada } \\
\hline Sim & $37(58,7)$ \\
\hline Não & $26(41,3)$ \\
\hline Tempo de perda de urina & $5,8 \pm 9,54 *$ anos \\
\hline \multicolumn{2}{|l|}{ Situação que perde urina } \\
\hline Ao tossir, espirrar, sorrir, colocar peso e/ou fazer esforço & $44(69,8)$ \\
\hline Precisa fazer xixi assim que sente vontade se não faz na roupa & $8(12,7)$ \\
\hline Apresenta as duas opções acima & $11(17,5)$ \\
\hline \multicolumn{2}{|l|}{ Queixa principal da consulta } \\
\hline Hemorragias & $4(6,3)$ \\
\hline Neoplasia & $4(6,3)$ \\
\hline Cirurgia ou retorno pós-cirurgia & $11(17,5)$ \\
\hline Problemas mamários & $3(4,8)$ \\
\hline Mostrar ou fazer exames & $9(14,3)$ \\
\hline Bexiga baixa e/ ou perda de urina & $22(34,9)$ \\
\hline Dor abdominal & $10(15,9)$ \\
\hline \multicolumn{2}{|l|}{ Número de gestações } \\
\hline Nenhuma & $7(11,1)$ \\
\hline De 1 a 3 & $26(41,3)$ \\
\hline De 4 a 5 & $17(27)$ \\
\hline Seis ou mais & $13(20,6)$ \\
\hline \multicolumn{2}{|l|}{ Aborto } \\
\hline Sim & $25(39,7)$ \\
\hline Não & $38(60,3)$ \\
\hline TOTAL & $63(100)$ \\
\hline
\end{tabular}

*Média \pm desvio padrão.

\section{Fatores de risco}

Quanto aos fatores de risco que podem estar associados à IU (Tabela 3), observando as respostas positivas, pode-se evidenciar que 1,6\% tinha doença neurológica; não houve nenhum caso de doença pulmonar obstrutiva crônica (DPOC); 28,6\% era diagnosticada com diabetes mellitus; 61,9\% com hipertensão arterial; 1,6\% era tabagista; 76,2\% fazia uso de cafeína; 82,5\% utilizava alguma medicação de uso continuo; 28,6\% usava antidiabético; 61,9\% fazia uso de anti-hipertensivos; 11,1\% era obesa; 34,9\% relatou problemas de constipação; 74,6\% já fez cirurgias pélvicas; e 23,8\% fez cirurgias abdominais. 
Tabela 3. Distribuição de participantes com incontinência urinária atendidas em um Hospital Universitário, segundo fatores de risco associados ( $n=63)$. Teresina (PI) - 2019.

\begin{tabular}{lc}
\hline Variáveis & $\mathbf{n}(\%)$ \\
\hline Doença neurológica & $1(1,6)$ \\
\hline DPOC & $0(0)$ \\
\hline Diabetes mellitus & $13(20,6)$ \\
\hline Hipertensão arterial sistêmica & $29(46)$ \\
\hline Tabagismo & $1(1,6)$ \\
\hline Cafeína & $48(76,2)$ \\
\hline Medicações & $14(22,2)$ \\
\hline Antidiabéticos & $18(28,6)$ \\
\hline Anti-hipertensivos & $39(61,9)$ \\
\hline Obesidade & $7(11,1)$ \\
\hline Constipação & $22(34,9)$ \\
\hline Cirurgias pélvicas & $47(74,6)$ \\
\hline Cirurgias abdominais & $15(23,8)$ \\
\hline
\end{tabular}

DPOC = Doença Pulmonar Obstrutiva Crônica

\section{DISCUSSÃO}

\section{Perfil sociodemográfico}

O estudo foi realizado apenas com pessoas que apresentavam IU e embora tenham ocorrido tentativas de coleta nos ambulatórios de ginecologia e urologia na busca por informações sobre ambos os sexos, o trabalho foi realizado apenas com mulheres. Acredita-se que entre os motivos estão o fato de as consultas no setor de ginecologia ocorrerem diariamente nos dois turnos e na urologia somente três vezes por semana, em um total de quatro atendimentos semanais, e que também atende as pessoas do sexo feminino. Outra razão é a melhor aceitação das mulheres em participar de pesquisa, e também por existirem mais mulheres acometidas por essa condição, além de ser o público que mais busca os serviços de saúde.

Pode-se afirmar que as mulheres utilizam mais os serviços de saúde do que os homens. Essa afirmação se justifica, em parte, pelas variações no perfil de necessidades de saúde entre os gêneros, sendo os principais motivos gravidez e parto, e o maior interesse das mulheres pela sua saúde ${ }^{12}$.

Quando analisada a idade, pode-se observar que a maioria apresenta uma idade média de 52 anos, o que corrobora a literatura a respeito do tema, demonstrando uma relação diretamente proporcional entre idade e IU, e esse aumento atinge seu nível máximo na meia idade, com prevalência de 30\% a 40\%, mantendo a elevação conforme aumenta a idade ${ }^{13}$. Em estudos sobre prevalência, apresentaram dados estatisticamente relevantes entre a faixa etária e a ocorrência de IU, demonstrando que a idade de forma isolada não provoca a incontinência, mas induz a modificações estruturais e funcionais no sistema urinário que predispõe ao problema ${ }^{8,14}$.

O estudo apresentou maior número de mulheres pardas com sintomas de IU, seguida de negras e brancas, o que corrobora a pesquisa que caracterizou a população piauiense, apresentando a cor parda como a mais prevalente, em 72,9\% da população. Reforça-se a isso um fato importante direcionado a cor da pele, o Brasil é um país miscigenado e tem a cor parda como predominante ${ }^{8,15}$.

Quanto à escolaridade, a maioria tinha pouco tempo na escola, dispondo apenas do ensino fundamental incompleto, mas houve uma distribuição razoável em todos os níveis de escolaridade. Em relação à ocupação, a maioria trabalhava em emprego fora ou em casa, seguida de aposentadas. A renda com percentual maior representou os participantes que ganhavam 
entre um e dois salários mínimos. Analisando o percentual de pessoas que são sustentadas com essa renda, a distribuição individual torna-se baixa.

De acordo com estudo feito pelo Instituto Brasileiro de Geografia e Estatística (IBGE), metade dos brasileiros adultos não frequentou a escola além do ensino fundamental, sendo que, aproximadamente, $35 \%$ não concluiu o ensino fundamental. O rendimento domiciliar per capita (por pessoa) do Brasil ficou em torno de $\mathrm{R} \$ 1.373 \mathrm{em} 2018$, sendo a renda do piauiense de $\mathrm{R} \$ 817$ por pessoa ${ }^{16}$. O que corrobora a característica apresentada nesta pesquisa, tendo a maioria dos participantes uma renda inferior à média brasileira. No entanto, não é possível afirmar nesse estudo a relação entre condição econômica e presença de incontinência.

\section{Perfil clínico}

A situação conjugal das entrevistadas mostrou que a maioria vive com um parceiro, casadas ou em uma união estável. Os achados demonstraram que mais da metade tem vida sexual ativa, entretanto pode ser percebido um número significativo de mulheres que não têm mais vida sexual ativa, mesmo tendo parceiro. A maior parte relatou que a IU não interfere na vida sexual e um pequeno percentual admite prejuízo nesse aspecto. Esse fato não invalida a importância do problema e a necessidade de intervenções junto a essas mulheres.

Um dos campos da vida afetado pela IU é a sexualidade, que é pouco abordado, mas envolve a subjetividade do ser humano em seus aspectos sociais, culturais, ideológicos, crenças e vivências, podendo ser afetada, acrescentado ao ato sexual em si, que ocorrendo uma abordagem tardia podem gerar consequências de uma abdicação completa da vida sexual ${ }^{17}$.

Sobre a preexistência de alguma doença, a maioria apresentou uma ou mais doenças além da incontinência. Enfermidades essas que na literatura demonstram fazer parte dos fatores de risco para o indivíduo desenvolver quadro de IU.

Quanto ao motivo de perda urinária, tiveram predomínio as perdas ocasionadas ao tossir, espirrar, sorrir, colocar peso e/ou fazer esforços, sendo esses sintomas mais relacionados à incontinência de esforço, o que corrobora os estudos sobre o tema, que teve a IUE como a mais prevalente em todos os estudos analisados ${ }^{4,7,8}$.

A IUE ocorre pelo aumento da pressão intra-abdominal sem a percepção prévia do desejo de urinar. Com o aumento da pressão abdominal e falha nos mecanismos da continência ao tossir, espirrar e levantar peso ocorre a perda de urina. A gravidade também é um fator relacional com a quantidade de perda urinária ${ }^{7}$.

O estudo se concentrou nos participantes que apresentavam sintomas de IU. Ao ser perguntado a respeito da queixa principal para a realização da consulta naquele dia no serviço, em torno de 1/3 relatou bexiga baixa e/ou perda de urina, o restante estava aguardando consulta para outros problemas e muitos, durante a entrevista, não sabiam ou não valorizavam o problema da IU como sendo algo a ser tratado, embora existisse o incomodo diante da condição apresentada.

$\mathrm{O}$ resultado se confirma com a literatura que mostra o constrangimento dos participantes ao falar sobre o assunto, levando uma a cada três pessoas que possuem incontinência a não procurar os serviços de saúde por esse motivo, reforçando as discussões sobre o assunto, sabendo-se que a maioria das vezes, as pessoas convivem com o problema por longos anos e avaliam como normal ${ }^{18}$.

Estudo sobre o tratamento de incontinência em mulheres idosas relata que a mulher tem a percepção da incontinência como sendo um processo natural do envelhecimento e, devido à falta de informação, por vergonha não busca o serviço de saúde para o tratamento da IU, utilizando, logo, fraldas ou absorventes como método de cuidado, o que é preocupante, pois pode interferir na vida sexual, nas atividades físicas, domésticas e no trabalho ${ }^{17,18}$.

Nos antecedentes obstétricos, pode-se observar o predomínio de múltiplas gravidezes e partos vaginais, correspondendo, respectivamente, a quase metade da primeira e a maioria da segunda. O somatório de mulheres que tiveram quatro partos ou mais representou um número importante e dessas, poucas realizaram algum parto cesário. Dessa forma, o estudo corrobora outros estudos que mostram o impacto da gravidez e, principalmente, do parto normal como um fator predisponente para a IU.

A pesquisa condiz com outros estudos de prevalência realizados que mostraram a gestação como fator de risco, desde a primeira gravidez, aumentando com as subsequentes, e que o parto normal aumenta a chance de desenvolver a incontinência. 
Em estudo populacional sobre IU na gestação, a prevalência de IU no período gestacional foi de 14,7\% em primíparas e a probabilidade de ocorrer a IU variou de forma significativa de acordo com as características das mulheres, como idade, peso e prática de exercícios físicos ${ }^{19}$. $\mathrm{E}$ com a ocorrência de múltiplas gravidezes aumenta ainda mais a predisposição à IU ${ }^{20}$. Em outra pesquisa pode ser observado que o risco de desenvolver IU é maior após o parto vaginal (71\%) do que após a cesariana, e a prevalência de IU maior que dez anos, quase triplicou após o parto vaginal em comparação com a cesariana ${ }^{21}$.

\section{Fatores de risco}

Vários são os fatores de risco sabidamente associados à IU, os quais foram investigados nesse estudo. Uma revisão integrativa sobre prevenção da IU em idosas reforça que além do avançar da idade, outros fatores podem predispor ao desenvolvimento da IU, que são: diabetes, obesidade, tabagismo, menopausa, doenças do sistema nervoso central, uso de medicamentos, restrição da mobilidade, múltiplos partos vaginais, impactação fecal, distúrbios psíquicos, diminuição do tônus da região perineal e uso de álcool ${ }^{21}$. E são esses fatores associados que aumentam a possibilidade de sua ocorrência.

No estudo, pode-se observar a existência de um ou mais desses fatores presentes nos participantes da pesquisa.

A hipertensão arterial foi identificada em quantidade expressiva no estudo, já a diabetes mellitus apareceu em uma proporção menor. Pode-se perceber no estudo que os pacientes com diabetes normalmente apresentam hipertensão e fazem uso de um ou mais medicamentos para uma ou ambas as enfermidades. Estudos sobre prevalência no climatério e de caracterização das mulheres incontinentes em ambulatório de ginecologia também não apresentaram, em seus resultados, relação significativa das duas enfermidades com a IU ${ }^{20,22}$.

Obteve-se um percentual de pessoas que fazem uso de cafeína e/ou que realizaram cirurgias pélvicas, seguido de problemas de constipação, cirurgias abdominais e obesidade/sobrepeso, em menor proporção. A cafeína é irritante vesical e produtos com presença da substância podem aumentar a necessidade de urinar. A constipação intestinal pode afetar a função urológica pela compressão da bexiga ao ocorrer estiramento do reto e pelo esforço feito na evacuação, que pode causar lesão na musculatura pélvica ${ }^{20}$. Cirurgias ginecológicas e episiotomia, podem ocasionar deformidades no assoalho pélvico, e o sobrepeso e a obesidade aumenta a pressão abdominal. Todos esses fatores podem predispor à perda involuntária de urina ${ }^{22}$.

Aponta-se a ocorrência de que a mesma participante apresenta um ou mais fatores de risco para o desenvolvimento de IU. Acredita-se que um fator isolado não leva à IU, mas, sim, a soma de fatores determinantes e condicionantes. O que leva a reforçar a multifatorialidade para a presença da IU.

Em relação às limitações, o estudo não teve amostra do sexo masculino, embora a proposta inicial fosse englobar os dois sexos. Isso se deveu ao fluxo reduzido de pacientes do sexo masculino, a não aceitação em participar da pesquisa, bem como ao tempo disponível para a conclusão do estudo, o que diminuiu a chance da realização de entrevista com ambos os sexos e impossibilitou o cruzamento desses dados. Outro fator que pode ser considerado limitante foi a realização do estudo somente com pessoas que relataram sintomas de IU, não permitindo a comparação dos dados entre pessoas sem e com IU.

Recomenda-se outros estudos sobre perfil no Brasil e Piauí, com um número amostral maior e com mais variáveis para obtenção de informações que possam subsidiar novas intervenções e implantação de serviços.

O estudo demostrou-se um importante contribuinte nas pesquisas sobre perfil, uma vez que reforça e incrementa informações sobre a temática e seus aspectos epidemiológicos. Pode-se observar também a importância de conhecer o perfil de pacientes incontinentes na instituição para que possam ser desenvolvidas estratégias para o cuidado e tratamento dessa clientela, assim como subsidiar novos trabalhos.

\section{CONCLUSÃO}

Com o estudo pode-se evidenciar que as participantes têm como características serem, em sua maioria, pardas, cor predominante nessa região do país. Com idade média de 52 anos e possuírem baixa escolaridade. A maioria dispõe de renda, contudo é responsável pelo sustento da família, o que torna o valor per capita baixo. 
O maior número de mulheres apresentou IUE, seguido de IUM e IUU, respectivamente. Em torno de 1/3 dessas mulheres buscaram o serviço do ambulatório para tratamento de disfunções miccionais, o que demonstra a existência de demanda para o tratamento de IU nessa instituição.

A maioria das entrevistadas teve múltiplas gestações, além de outros fatores de risco que apareceram de forma significativa no estudo, que foram hipertensão arterial, uso contínuo de medicações, em especial anti-hipertensivos, e já realizaram cirurgia pélvica. Em menor proporção, mas ainda com certa predominância, apareceram pessoas com diabetes e em uso de antidiabético, com problemas de constipação e que já realizaram cirurgia abdominal.

Dessa forma, é possível concluir que todas as mulheres do estudo apresentaram mais de um fator de risco para desenvolver IU, reforçando sua multifatorialidade, bem como a importância de um tratamento holístico e multiprofissional.

Quando ressaltado o objetivo de conhecer o perfil dos pacientes, embora com resultado amostral pequeno, diante da estimativa da população com essa disfunção, apresentou resultados satisfatórios e condizentes com a literatura existente, uma vez que os dados referentes à idade, escolaridade, renda familiar e raça/cor, bem como antecedentes obstétricos e fatores de risco associados corroboram com estudos nacional e internacional realizados sobre o tema.

O número de mulheres que têm sua vida sexual com algum grau de interferência devido a IU, mesmo em quantidade não muito expressiva nesse estudo, deve ser valorizado, assim como a negligência na busca do tratamento por desconhecimento do problema e/ou da possibilidade de tratar.

\section{CONTRIBUIÇÃO DOS AUTORES}

Conceitualização: Braga FCSAG e Benício CDAV; Metodologia: Braga FCSAG e Benício CDAV; Investigação: Braga FCSAG, Silva A, Costa AQe Santos ES; Redação - Primeira versão: Braga FCSAG e Siqueira RMOT; RedaçãoRevisão \& Edição: Braga FCSAG, Benício CDAV e Bezerra SMG; Recursos: Braga FCSAG; Supervisão: Braga FCSAG.

\section{DISPONIBILIDADE DE DADOS DE PESQUISA}

Dados serão disponibilizados mediante pedido.

\section{AGRADECIMENTOS}

Agradecemos a todos os participantes da pesquisa, sua contribuição foi essencial para atingirmos nossos objetivos. Obrigada.

\section{REFERÊNCIAS}

1. Brasil DM, Nicolau Al, Bilhar AP, Karbage AS, Lucena SV, Carmo TF et al. Incontinência urinária e função sexual feminina: revisão integrativa de questionários validados. Acta paul. Enferm. 2018; 31(5): 558-563. https://doi.org/10.1590/1982-0194201800077

2. Sociedade Portuguesa De Ginecologia. Consenso Nacional sobre Uroginecologia Incontinência Urinária de Esforço: Um perfil da clientela, junho de 2018 - Secção Portuguesa de Uroginecologia.

3. Riemsma R, Hagen S, Kirschner-Hermanns R, Christine N, Helle W, Karl-Erik A et al. A incontinência pode ser curada? Uma revisão sistemática das taxas de cura. BMC Med.2017; 15: 63. https://doi.org/10.1186/s12916-017-0828-2

4. Benício CDAV, Luz MHBA, Carvalho NV, Brito BAM, Ferreira JLS. Conhecimento de Mulheres Incontinentes sobre Incontinência Urinária: uma Reflexão Teórico Crítica. Estima-Brazilian Journal of Enterostomal Therapy. 2017; 15(1): 58-61. https://doi. org/10.5327/Z1806-3144201700010010

5. Junqueira JB, Santos VLCG. Incontinencia urinaria en pacientes hospitalarios: prevalencia y factores asociados. Rev. Latino-Am. Enfermagem [Internet]. 2017; 25: e2970. https://doi.org/10.1590/1518-8345.2139.2970

6. Abrams P, Cardozo L, Wagg A, Wein A. Incontinence. 6th International Consultation on Incontinence. International Contnence Society. Tokyo. 2017. 
7. Santos CRS, Santos VLCG. Prevalência da incontinência urinária em amostra randomizada da população urbana de Pouso Alegre, Minas Gerais, Brasil. Rev. Latino-Am. 2010;18(5): 1-8. http://doi.org/10.1590/S0104-11692010000500010

8. Benício CDAV, Luz MHBA, Lopes MHBM, Carvalho NAR. Incontinência Urinária: Prevalência e Fatores de Risco em Mulheres em uma Unidade Básica de Saúde. Estima-Brazilian Journal of Enterostomal Therapy. 2016; 14 (4): 161-168. https://doi. org/10.5327/Z1806-3144201600040002

9. Oliveira LGP, Oliveira AG de, Souza G de, Resende MA. Incontinência urinária: a atuação do profissional de enfermagem. REAS. 2018; (18):e118. https://doi.org/10.25248/reas.e118.2019

10. Benício CDAV. Avaliação do impacto de blog na autoconfiança de pacientes e cuidadores praticantes do cateterismo intermitente limpo. Tese (Doutorado em Enfermagem) - Universidade Federal do Piauí, Centro de Ciências da Saúde, Programa de PósGraduação em Enfermagem, Teresina, 2018.

11. Favoretto NB. Development of a virtual forum for people with spina bifida and their families: Collaboration and Exchange of Technology between Brazil and Germany. 2015. Tese (Doutorado em Enfermagem) - Universidade de São Paulo, Faculty of rehabilitation Sciencesat the University of Dortmund, Germany, São Paulo, 2015.

12. Silva SLA, TorresJL, Peixoto SVA. Fatores associados à busca por serviços preventivos de saúde entre adultos brasileiros: Pesquisa Nacional de Saúde, 2013. Ciênc. saúde coletiva. 2020; 25(3):783-792. https://doi.org/10.1590/1413-81232020253.15462018

13. Bitencourt GR, Felippe NHMD, Santana RF. Diagnóstico de enfermagem eliminação urinária prejudicada em idosos no pósoperatório: um estudo transversal. Rev. enferm. UERJ.2016; 24(3):e16629. https://doi.org/10.12957/reuerj.2016.16629

14. Townsend MK, Curhan GC, Grodstein F, Resnick NM. Original research: rates of renission improvement and progression of urinary incontinence in Asian, Black and White womwn, AJN The American Journal of Nursing, 2011;111(4):34-35. https://doi. org/10.1097/01.NAJ.0000396551.56254.8f

15. Instituto Brasileiro De Geografia E Estatística (IBGE). [acesso em 23 de maio de 2019] Disponível em: https://agenciadenoticias. ibge.gov.br/agencia-sala-de-imprensa/2013-agencia-de-noticias/releases/23852-ibge-divulga-o-rendimento-domiciliar-percapita-2018

16. Pacagnella RC, Martinez EZ, Vieira EM. Validade de construção de uma versão em português do Índice de Função Sexual Feminina. Cafajeste. Saúde Pública. 2009; 25 (11): 2333-2344. https://doi.org/10.1590/S0102-311X2009001100004

17. Oliveira JR, Garcia RR. Cinesioterapia no tratamento da incontinência urinária em mulheres idosas. Rev. bras. geriatr. gerontol. 2011; 14 (2): 343-351. https://doi.org/10.1590/S1809-98232011000200014

18. Matos MAB, Barbosa BLA, Costa MC, Rocha FCV, Almeida CAPL, Amorim FCM. As Repercussões Causadas pela Incontinência Urinária na Qualidade de Vida do Idoso. Rev Fund Care Online.2019; 11(3):567-575. https://doi.org/10.9789/2175-5361.2019. v11i3.567-575

19. Ting HY, Cesar JÁ. Urinary incontinence among pregnant women in Southern Brazil: A population-based cross-sectional survey. PLOS ONE. 2020; 15(6): e0234338. https://doi.org/10.1371/journal.pone.0234338

20. Mourão LF, Araújo Luz MHB, Marques ADB, Vasconcelos Benício CDA, Teixeira Nunes BMV, Pereira AFM. Caracterização e fatores de risco de incontinência urinária em mulheres atendidas em uma clínica ginecológica. Estima-Brazilian Journal of Enterostomal Therapy. 2017; 15(2). https://doi.org/10.5327/Z1806-3144201700020004

21. Milsom I, Gyhagen M. The prevalence of urinary incontinence, Climacteric, 2019; 22(3): 217-222. https://doi.org/10.1080/1369 7137.2018 .1543263

22. Oliveira TM, Valdez FML, Lima KES, Magalhães MS, Abdon APV, Bezerra IN. Prevalência de Incontinência Urinária e Fatores Associados em Mulheres no Climatério em uma Unidade de Atenção Primária à Saúde. Rev Bras Promoç Saúde, 2015; 28(4): 606-612. Disponível em: https://periodicos.unifor.br/RBPS/article/view/5422/pdf 